\title{
Multilinguales
}

\section{Multilinguisme et injustice sociale linguistique au}

Botswana

Multilingualism and linguistic social injustice in Botswana

\section{Kagiso Jacob Sello}

\section{(2) OpenEdition}

\section{Journals}

\section{Édition électronique}

URL : http://journals.openedition.org/multilinguales/4012

DOI : $10.4000 /$ multilinguales. 4012

ISSN : 2335-1853

\section{Éditeur}

Université Abderrahmane Mira - Bejaia

\section{Référence électronique}

Kagiso Jacob Sello, « Multilinguisme et injustice sociale linguistique au Botswana », Multilinguales [En ligne], 11 | 2019, mis en ligne le 17 juillet 2019, consulté le 01 juin 2020. URL : http:// journals.openedition.org/multilinguales/4012; DOI : https://doi.org/10.4000/multilinguales.4012

Ce document a été généré automatiquement le 1 juin 2020

\section{cc)}

Multilinguales est mise à disposition selon les termes de la Licence Creative Commons Attribution Pas d'Utilisation Commerciale - Pas de Modification 4.0 International 


\title{
Multilinguisme et injustice sociale linguistique au Botswana
}

\author{
Multilingualism and linguistic social injustice in Botswana
}

\author{
Kagiso Jacob Sello
}

1 Parmi toutes les formes possibles qui freinent la participation des minorités linguistiques à la vie sociale, la forme linguistique est la moins évidente et la plus silencieuse. Dans un sens général, le terme de langue minoritaire, selon la Charte européenne des langues régionales ou minoritaires (1992: p. 2), renvoie à des langues autochtones :

pratiquées traditionnellement sur un territoire d'un Etat par des ressortissants de cet Etat qui constituent un groupe numériquement inférieur au reste de la population de l'Etat; et différentes de la (des) langue(s) officielle(s) de cet Etat; elle n'inclut ni les dialectes de la (des) langue(s) officielle(s) de l'Etat ni les langues des migrants.

2 La majorité des langues minoritaires sont également des langues régionales dans le sens où elles sont parlées sur une partie limitée du territoire d'un État où elles peuvent, dans certaines situations, être les langues les plus parlées. Autrement dit, le terme minoritaire renvoie à un critère numérique alors que "régional " est lié à l'aspect géographique (Sello, 2013). Et souvent, un nombre important des personnes issues des minorités linguistiques parlent approximativement voire pas du tout les langues officielles de l'État (Chebanne, 2015).

3 Dans le contexte botswanais, on observe un manque de données sur la variable linguistique et ses impacts potentiels sur la prestation de services. Il existe certes des anecdotes mais pas suffisamment de données linguistiques probantes sur lesquelles se fier lorsqu'on veut démontrer en quoi et comment la langue devient un déterminant majeur dans la participation à la vie sociale des individus issus des minorités linguistiques. Il n'est donc pas surprenant de constater que, souvent, lorsqu'on s'interroge sur la non-participation à la vie sociale des minorités linguistiques, on se limite à l'origine ethnique, la religion, le statut socio-économique ou la culture (LaVeist, 2005; Williams et Collins, 2001). Peu d'études questionnent le rôle de la 
langue. Or, la langue joue un rôle majeur dans la participation sociale et par voie de conséquence dans l'accès aux services de l'État, ainsi qu'à la qualité de ces services. Pour pallier ce manque de données probantes, nous avons bon espoir que cet article donnera suite à une recherche plus approfondie sur le sujet.

4 Cette présentation vise à démontrer que, dans le contexte botswanais, les individus issus de minorités linguistiques sont privés de leurs droits en termes d'accès aux services publics lié à l'utilisation des langues et subissent par conséquent une injustice sociale. Dans un premier temps, nous nous intéresserons brièvement à la politique linguistique du Botswana. Ceci nous permettra de faire le lien entre la politique linguistique du pays et la justice sociale liée à l'utilisation des langues, notion que nous définirons dans un deuxième temps. Nous tâcherons ensuite de démontrer comment la langue devient un déterminant de la justice sociale. Nous formulerons quelques propositions susceptibles d'améliorer l'accès des groupes minoritaires aux services publics avant de conclure.

\section{La politique linguistique du Botswana}

5 Si le Botswana présente une situation linguistique moins complexe que la plupart des pays d'Afrique subsaharienne, dont certains comptent plus de 200 langues, ce pays n'en est pas moins caractérisé par la coexistence de plusieurs langues sur son territoire. En effet, il compte en tout vingt-neuf langues qui se répartissent en trois groupes linguistiques distincts (Hasselbring, 2000a, 2000b ; Janson, 2000 ; Janson \&Tsonope, 1991). Le groupe bantou, qui constitue le groupe majoritaire, comptabilise quatorze langues: setswana, shekgalagari, ciikuhane, shiyeyi, ikalanga, setswapong, sebirwa, otjiherero, thimbukushu, zezuru, silozi, rugciriku, nambya, etisindebele. Le groupe khoisan est au deuxième rang, avec douze langues, dont trois langues san : !xóõ, \#hua, etju|'hoansiet neuf langues khoe : |Gwi, \|Gana, Khwedam, nama, naro, shua (Cire-Cire), tshwa, kuaet $\neq$ Kx'au\|'ein (Monaka et al., 2016) Deux langues, l'afrikaans et l'anglais, constituent le groupe germanique. La langue de signe botswanaise complète la liste. La coexistence de toutes ces langues sur son territoire fait donc du Botswana un pays multilingue. Dans les textes, l'État botswanais adhère à l'idée de multilinguisme. Ainsi, la politique culturelle nationale du Botswana (National Cultural Policy, 2001) met l'accent sur la diversité multilinguistique et multiculturelle du pays. Les langues sont considérées comme porteuses de l'héritage culturel et à ce titre doivent être développées à travers la recherche et la documentation. Cette politique prévoit également la mise en œuvre des stratégies visant à développer et encourager l'utilisation des diverses langues autochtones au niveau local afin de faciliter la cohésion nationale. Dans les faits, il n'en est pas ainsi.

6 Selon Chebanne et Creissels (2001), la majorité des textes émanant des organismes officiels ou semi-officiels résument la politique linguistique du Botswana ainsi: l'anglais est la langue officielle et le setswana la langue nationale. Les auteurs ont également remarqué que pour certains organismes, les deux langues sont co-officielles. Toutes les autres langues sont considérées comme des langues minoritaires, auxquelles il n'est fait aucune allusion dans la politique linguistique du Botswana . Les débats sur ces langues provoquent souvent des réactions plutôt négatives de la part des autorités. En effet, la reconnaissance de ces langues est perçue comme une menace pour l'unité 
nationale qui fait la cohésion et la fierté du pays. Cette vision est cohérente avec l'analyse de Baker, selon qui

entretenir les minorités linguistiques et la diversité linguistique pourrait avoir pour conséquence moins d'intégration, moins de cohésion, davantage d'antagonisme et de conflits dans la société. » (1993 : p. 248).

Par voie de conséquences, seuls l'anglais et le setswana sont utilisés dans la prestation de services publics par le gouvernement, créant ainsi un contexte d'injustice sociale envers les groupes minoritaires.

\section{Justice sociale linguistique}

Il n'est jamais aisé de définir un terme à la signification aussi étendue que celui de justice sociale car cette notion est née de la conjugaison de divers facteurs. La justice sociale renvoie à ce qui est socialement accepté comme juste. La justice sociale est fondée sur l'égalité des droits pour tous les peuples et la possibilité pour tous les êtres humains, sans discrimination, de bénéficier du progrès économique et social du pays. Jean-Fabien Spitz reformule la définition que John Rawls donne à cette notion de la manière suivante :» un ensemble institutionnel est juste si les règles en sont disposées de manière à fonctionner à l'avantage de l'ensemble des citoyens et non pas d'une partie de ceux-ci » (2011: p. 56). La justice sociale est donc une construction morale et politique. Elle est souvent définie en termes économiques en tant que distribution juste et équitable des richesses entre les différents membres de la société. Cela pourrait s'expliquer en partie par le fait que, dans sa constitution, l'Organisation Mondiale du Travail présentait expressément la justice sociale comme un constituant clé pour établir une paix universelle et durable alors que nulle mention n'en est faite ni dans la Déclaration universelle des droits de l'homme, ni dans les pactes internationaux relatifs aux droits de l'homme.

9 Néanmoins, le terme de justice sociale apparait récemment à plusieurs reprises accolé au terme de développement social dans la Déclaration de Copenhague, publiée à l'issue du Sommet mondial pour le développement social en 1995. Dans cette déclaration, il est question de soutenir les aspirations de justice sociale des groupes minoritaires et de leur garantir un environnement leur permettant de participer pleinement à la vie sociale, économique et politique de leurs pays. Donc, le mot clé de la justice sociale est la participation. Mais alors, comment la langue favorise ou au contraire freine-t-elle la participation sociale des minorités linguistiques? Comment devient-elle un des déterminants de la justice sociale?

10 Pour répondre à cette question, il convient de parler de l'utilisation des langues dans la sphère publique. Nous avons précédemment indiqué que, dans les textes, l'État botswanais adhère à l'idée du multilinguisme en reconnaissant la diversité des langues en présence sur le territoire et prévoit que les citoyens puissent avoir accès aux services dans leur propre langue et être protégés de toute discrimination. Cela signifie que, théoriquement, toutes les personnes qui se rendent au commissariat de police pour déclarer un vol par exemple, devraient pouvoir faire leur déclaration dans la langue de leur choix. De même, l'hôpital public devrait pouvoir offrir des services de soins dans la langue que préfèrent les patients. Or, dans le contexte actuel botswanais, que ce soit au commissariat ou à l'hôpital ou tout autre service public, le service n'est offert qu'en anglais ou en setswana. Ainsi, le fait de ne pas pouvoir obtenir de services 
dans la langue dans laquelle le demandeur du service se sent le plus à l'aise créé un obstacle à la communication et constitue ce que nous appelons une injustice sociale linguistique. En effet, pour participer pleinement, il faut pouvoir communiquer avec les autres participants avec aisance. Ceci requiert le partage et une relative maitrise d'une langue commune, sans laquelle toute communication serait difficile, voire impossible. On pourrait catégoriser les principaux obstacles linguistiques qui entravent la participation sociale des minorités linguistiques comme suit : l'accès à l'information sur les services, le recours aux services, l'accès aux services ainsi que la qualité des services.

\section{Accès à l'information sur les services}

11 Le premier obstacle à la participation des groupes minoritaires à la vie sociale est celui de l'accès à l'information sur les services offert par l'État. Lorsqu'une information doit être diffusée parmi la population concernée (par exemple au sujet de la propagation d'une maladie chronique, la recommandation d'une nouvelle technique ou d'une innovation agricole ou encore concernant la circulation des faux billets sur le marché), le gouvernement botswanais utilise la radio, la télévision, les affiches publiques ou le service de sms pour communiquer avec les citoyens. L'information est relayée en anglais ou en setswana. Aucun cadre juridique ne favorise la diffusion d'informations dans les langues minoritaires, excluant ainsi ces groupes. De même, minorités linguistiques sont exclus des études ou enquêtes sur les services et des plans de vulgarisation étant donné que ces actions ne se font qu'en anglais ou qu'en setswana. Par conséquent, la barrière linguistique est la cause non seulement d'une mauvaise pénétration des campagnes de promotion des services auprès des minorités linguistiques qui n'ont pas accès à l'information mais de surcroit, ils ignorent également quels services leurs sont proposés et accessibles.

\section{Recours aux services}

Des études, en particulier celles entreprises au Canada, ont démontré que la langue a une incidence quant au recours aux services (Gagnon-alpin et al. 2014, Bouchard et Martin. 2013).Les résultats de ces recherches menées dans le domaine de la santé, peuvent être étendus à d'autres domaines et à d'autres pays. Ainsi, selon ces études, la première difficulté se présente au moment où la personne qui a besoin d'un service doit décider de solliciter ou non ce service. Ces études ont ainsi mis en lumière le fait que des individus ne parlant pas suffisamment voire pas du tout les langues de communication employées pour offrir le service estiment qu'ils auront des difficultés à se faire comprendre ou à comprendre les prestataires de services. La non maîtrise de la langue du service installe ainsi le doute chez les personnes désireuses de demander un service. En raison de cette insécurité linguistique, ces personnes n'osent pas toujours faire face à cet obstacle linguistique et renoncent souvent à demander les services.

\section{Efficacité de la communication et qualité du service}

13 Si la décision de recourir aux services est prise, les barrières linguistiques ne sont pas pour autant abattues. En effet, parler une langue ne signifie pas pour autant qu'on est capable de la parler à bon escient, d'autant plus lorsque l'aspect émotionnel entre en 
jeu comme par exemple lorsque le médecin vient de vous annoncer que vous êtes atteint d'une maladie incurable ou lorsque le temps est un facteur de risque, après un accident de travail ou de circulation. L'efficacité de la communication en est alors affectée et par conséquent la qualité du service également. Ainsi, le choix de la langue ou les langues d'administration d'un État semble traduire une inégalité sociale d'accès aux ressources et contribuent de ce fait à la promotion de l'injustice sociale. Mais, l'injustice sociale, est-elle insurmontable? Nous répondons par la négative à cette question, ce qui nous conduit à formuler la recommandation suivante relative aux stratégies à adopter par les pouvoirs publics pour améliorer la situation.

\section{Recommandations}

14 L'État doit accorder aux langues minoritaires le statut de langues de travail au même titre que l'anglais et le setswana dans les régions où ces langues sont majoritaires. De cette recommandation découlent les propositions suivantes :

\section{Première proposition : le recours à des employés trilingues}

15 L'État devra faire un effort d'employer dans les services publics, des personnes maitrisant la langue minoritaire de la région où ils exercent en plus des deux langues officielles. Ces personnes seraient sélectionnées non seulement sur la base de leur trilinguisme, mais plus important encore, sur la base de leur compétence professionnelle spécifique au domaine du service à offrir. Cette compétence professionnelle se caractériserait par la connaissance approfondie du service à offrir, des connaissances relatives à la langue comme déterminant de la prestation du service, à la situation socioéconomique et culturelle des communautés en situation minoritaire et aux caractéristiques du travail en contexte linguistique minoritaire. Cette proposition nous semble la plus adaptée car le fait que l'usager partage les mêmes langue et culture avec le prestataire de service rend la communication verbale et non verbale plus directe, efficace, l'usager étant en situation de sécurité linguistique. Néanmoins, elle nous semble la plus difficile à mettre en œuvre.

\section{Deuxième proposition : la publication dans les langues minoritaires}

Le fait de donner un statut « officiel » aux langues régionales inciterait également les pouvoirs publics dans les régions concernées à développer l'orthographe de ces langues, à en formaliser la grammaire et à les moderniser. On entend par modernisation le fait d'adapterla langue aux besoins linguistiques de la société émergeants, surtout au niveau du lexique (Weiner, 1966 ; Furgeson, 1968 ;Agyekum, 2008). Les régions seraient chargées de produire des documents informatifs dans ces langues. Ainsi, la littératie des personnes issues de ces minoritaires serait alors renforcée, améliorant de ce fait l'accès à l'information, l'impact des campagnes de promotion de services et un usage accru des services proposés. 


\section{Troisième proposition : les cours de langues aux prestataires de services}

La publication de documents et matériels éducatifs en langues minoritaires pourrait en outre faciliter l'enseignement de ces langues aux prestataires de services qui seraient affectés dans les régions concernées.

\section{Quatrième proposition : les services d'interprétariat}

Cette solution est de loin la plus adoptée dans les pays soucieux de garantir un accès aux services aux minorités linguistiques mais elle n'est pas pour autant la plus satisfaisante dans l'état actuel. En effet, dans la plupart de ces pays, les interprètes n'ont pas de codes d'éthiques, alors que certains services requièrent le secret professionnel. En plus, dans beaucoup de pays, l'interprétariat est souvent réduit au bilinguisme. Alors, quand le besoin d'un service d'interprétariat se fait sentir, on fait appel aux membres de la famille, à des amis ou même des employés du service concerné du moment où ils ont eu contact, aussi minime soit-il, avec la langue du demandeur du service. Même lorsqu'on fait appel à des interprètes professionnels, ils ne sont pas formés à intervenir dans un milieu linguistique minoritaire. Pour que cette mesure soit efficace, le l'État devrait veiller à ce que les interprètes acquièrent les compétences relatives au service à offrir, a l'interprétariat général ainsi qu'à l'interprétariat en contexte minoritaire. En effet, la compétence interprétative est une compétence qui doit être reconnue au même titre que la compétence professionnelle ou technique.

Pour conclure cette présentation, rappelons que le droit botswanais ne reconnait explicitement nulle part un «droit aux services ». L'État n'est donc pas juridiquement obligé de donner un accès universel et gratuit aux services qu'il propose. C'est un choix politique. Néanmoins, l'État a une obligation morale de ne pas discriminer des personnes en fonction de leur profil linguistique. Donc, outre la reconnaissance de la diversité linguistique de son territoire, l'État doit également veiller à ce que tous les services publics soient offerts à toutes les personnes sans aucune discrimination, y compris celle de nature linguistique. L'État doit prendre conscience des exigences imposées par la communication interlinguistique dans les services publics. Il est de son devoir d'œuvrer à la facilitation de l'accès à l'information des personnes ne maitrisant pas les langues officielles, leur accueil dans les centres de services publics ainsi qu'à la qualité des services qui leur sont offerts.

Le gouvernement botswanais montre des signes d'amélioration en ce sens. Récemment, le maire adjoint de la ville de Francistown au nord-est du pays inaugurait un centre spécialisé dans le dépistage du VIH chez des personnes sourdes et malentendantes dans le but de faciliter leur accès au service de santé (Mkhutshwa, 2018). En effet, il avait été observé que cette population rencontrait de difficultés pour accéder aux services de santé notamment à cause du manque de personnel de santé pouvant communiquer efficacement en langue de signes botswanaise. C'est ainsi que le personnel employé dans ce centre est formé à la langue de signes botswanaise et au dépistage du VIH. Les autorités prévoient également de traduire les termes médicaux en langue de signes. Nous avons bon espoir que cette initiative prometteuse sera prochainement étendue à la vaste mosaïque des langues minoritaires qui composent le paysage linguistique botswanais. 


\section{BIBLIOGRAPHIE}

AGYEKUM,K, «Lexical expansion and elaboration in Akan : Afisem and the media », dans S. G. Obeng and B.A.S Hartford (dirs.),Political Discourse Analysis, New York : Nova Science Publishers Inc, 2008, pp. $73-88$.

BAKER,C.Foundations of Bilingual Education and Bilingualism.Clevedon, UK/Philadelphia, PA : Multilingual Matters, 1993.

BOUCHARD, L. et MARTIN, D, « Les minorités linguistiques du Canada et la santé », Healthcare Policy/ Politiques de santé, numéro spécial, vol. 9, Toronto, Canada, 2013, pp. 38-47.

CHARTEEuropéenne des Langues Régionales ou Minoritaires, 1992. Disponible sur CELRM. Consulté le $14 / 02 / 2019$.

CHEBANNE, A, « Negative Multicultural Consequences of Botswana Restrictive Language Policy in Education : Mourning the loss of Khoisan Languages », dans A. Jotia\& D. Jankie (dir(s)) Multicultural Education Discourses : Breaking Barriers of Exclusion in selected African Contexts. Zebra Publishing. Windhoek, Namibia,2015, Chapter 1, pp 5-24.

CHEBANNE, A. et CREISSELS,D. « Les langues et la politique linguistique du Botswana », dans Compagnon, Daniel \&Mokopakgosi, Brian T, (dirs), Le Botswana contemporain. Paris : Karthala, 2001, pp. 332-354.

FERGUSON, C. A. « Language development. » dans J. A. Fishman, C. A. Ferguson and J. Das Gupta (dirs.),Problems of Developing Nations,New York : Wiley : 1968, pp. 27-36.

GAGNON-ARPINI, et al,» Accès et utilisation des services en langue minoritaire », dans Rodrigue Landry (dir.), La vie dans une langue officielle minoritaire au Canada, Quebec, Presses de l'Universite Laval, 2014, pp. 193-219.

HASSELBRING,S.A sociolinguistic survey of the languages of Botswana, vol. 1. Sociolinguistic studies of Botswana language series. Gaborone : Basarwa Languages Project, under the University of Botswana/University of Tromsø Collaborative Basarwa Research Programme. 2000a

HASSELBRING, S.» Where are the Khoesan of Botswana? », dansBatibo, Herman, M. \&Smieja, Birgit (dirs), Botswana :The Future of Minority Languages,Frankfurt/Main : Peter Lang, 2000b, pp. 13-32.

JANSON,T. etTSONOPE,J,Birth of a National Language - The History of Botswana,Gaborone : Heinemann, 1991.

LAVEIST,T. A, « Disentangling race and socioeconomic status : A key to understanding health inequalities. " Journal of Urban Health : Bulletin of the New York Academy of Medicine, 82, 2005, pp 26-34.

MKHUTSHWA,L. Health care discriminates against the deaf. (2018, October 25). Disponible surhttp:// www.mmegi.bw/. Consulté le 24/02/2019

MONAKA,K. C. ; MOUMAKWA,V. T. et MOTHEIS,» The training of teacher of African languages for Primary Schools in Botswana. » Linguistics Association for Southern Africa, Volume 4, No. 4, 2016, pp. 51-64

NATIONAL POLICY ON CULTURE, Ministry of Labourand Home Affairs, Department of Culture and Youth. 2001 
RAWLS,J, A Theory of Justice, Cambridge,MA : Harvard University Press,1971.

SELLO, K. J,Analyse matricielle définitoire : Outil linguistique au service de la conception d'un programme de traduction pour des étudiants de langues étrangères (Thèse non publiée, Université Paris Sorbonne IV), 2013.

SPITZ, J-F, « John Rawls et la question de la justice sociale », Études 2011/1 (Volume 414), 2011, pp. 55-65.

UNITED NATIONS, International Covenantson Human Rights. 1966. Disponible sur ICHR.Consulté le $14 / 02 / 2019$

UNITED NATIONS,International LabourOrganisation. 1919. Disponible sur ILO. Consulté le $14 / 02 / 2019$.

UNITED NATIONS,Universal Declaration of Human Rights. 1948. Disponible sur UDHR.Consulté le $14 / 02 / 2019$.

UNITED NATIONS,World Summit for Social Development. 1995. Disponible sur WSSD.Consulté le $14 / 02 / 2019$.

WEINER,M.Modernization : The Dynamics of Growth, New York and London : Basic Books, 1966.

WILLIAMS,D. R. etColLINS,C,» Racial residential segregation : A fundamental cause of racial disparities in health ", Public Health Reports, 116 (5), New York : SAGE, 2001, pp 404-416.

\section{RÉSUMÉS}

L'État botswanais adhère à l'idée du multilinguisme. Or dans les faits, rien n'est fait pour mettre en pratique cette reconnaissance. Seuls l'anglais et le setswana sont utilisés dans la prestation des services publics. Par conséquent, les minorités linguistiques subissent une injustice sociale linguistique. L'article proposera quelques pistes de réflexion.

Botswana adheres to the idea of multilingualism. However, nothing is being done to give effect to this recognition. Only English and Setswana are used for the provision of public services by the government. As such, members of linguistic minorities are victims of language related social injustice. This article will propose some directions for reflection.

\section{INDEX}

Keywords : multilingualism, social justice, public services, linguistic minorities, Botswana

Mots-clés : multilinguisme, justice sociale, services publics, minorités linguistiques, Botswana

\section{AUTEUR}

KAGISO JACOB SELLO

University of Botswana, Botswana 